\title{
Network of Fuzzy Comparators for Ovulation Window Prediction
}

\author{
Łukasz Sosnowski ${ }^{1(\bowtie)}$, Iwona Szymusik ${ }^{2}$, and Tomasz Penza ${ }^{3}$ \\ 1 Systems Research Institute, Polish Academy of Sciences, Newelska 6, \\ 01-447 Warsaw, Poland \\ sosnowsl@ibspan.waw.pl \\ 2 Department of Obstetrics and Gynecology, Medical University of Warsaw, \\ Żwirki i Wigury 61, 02-091 Warsaw, Poland \\ iwona.szymusik@gmail.com \\ 3 OvuFriend Sp. z o.o., Złota 61/100, 00-819 Warsaw, Poland \\ tomasz.penza@ovufriend.com
}

\begin{abstract}
This paper presents the problem and the solution of ovulation date prediction based on simple data acquired by a woman in home environment. It describes a method of processing collected data as a multivariate time series. The novelty of this algorithm lies in its ability to predict the ovulation date and not only to retrospectively detect it. This is achieved by applying the fuzzy network of comparators (NoC) to compare the menstrual cycle being analyzed with the reference set of historical cycles.
\end{abstract}

Keywords: Ovulation prediction $\cdot$ Fertility $\cdot$ Network of comparators $\cdot$ Compound objects $\cdot$ Time series data $\cdot$ comparators $\cdot$ Similarity $\cdot$ Fuzzy sets $\cdot$ Classifiers

\section{Introduction}

Infertility is one of the most challenging contemporary problems as the population is aging. The procreation window of a woman is constantly being shifted towards the age of about 35 years. It differs slightly across the world, but the tendency is uniform. Statistics say that every fifth couple that is trying to conceive (TTC) has a problem to achieve pregnancy in the first 12 months of efforts [1]. This effect can be reinforced by polluted environment, frequent travels, stress or an unhealthy lifestyle, all of which may affect the length of the fertility window.

In this situation, the ability to precisely determine the date of ovulation becomes crucial. Knowing the date of ovulation allows the couple to plan their intercourses (in the immediate vicinity of ovulation) in order to maximize

Co-financed by the EU Smart Growth Operational Programme 2014-2020 under the project "Development of New World Scale Solutions in the Field of Machine Learning Supporting Family Planning and Overcoming the Infertility Problem", POIR.01.01.0100-0831/17-00.

(C) Springer Nature Switzerland AG 2020

M.-J. Lesot et al. (Eds.): IPMU 2020, CCIS 1239, pp. 800-813, 2020.

https://doi.org/10.1007/978-3-030-50153-2_59 
chances of successful conception. In case of an anovulatory cycle, the knowledge that it is anovulatory reduces the stress associated with waiting for possible pregnancy confirmation and allows women to concentrate on better preparation for their next cycle. Accurate designation of the ovulation day may also reduce some equally important problems on the male side.

From the medical point of view, assessing the Graafian follicle using ultrasound (its presence, size and proof of rupture) and measuring the serum progesterone concentration (it should be over $5 \mathrm{ng} / \mathrm{ml}$ in the luteal phase) are the only reliable methods that are considered to prove that ovulation has occurred. In natural family planning there are other methods that are less reliable, but they nonetheless allow couples to obtain some information on ovulation at home. One of them is measuring the basal body temperature (BBT). An increase in $\mathrm{BBT}$ of at least $0.2^{\circ} \mathrm{C}$ above the baseline taken at the same time every morning indicates that ovulation has occurred $[8,9]$. Additionally, the measurements have to be taken in the same body area and before performing any activities (ideally immediately after waking up). Moreover, it is required to measure BBT using the same device everyday. These limitations can impose great difficulties and sometimes cannot be accepted by women trying to conceive. It also requires a lot of determination. Another method is analyzing the variability of cervical mucus (the amount and consistency of cervical secretions) [3,9]. This method is also associated with various inconveniences related to measurements. The observation of the uterine cervix position, texture and opening constitute the third possibility in this field [9]. However this method requires a lot of experience and knowledge of one's own anatomical structure. It is very individual and not every woman will be able to reliably make the appropriate measurements. All these three methods belong to the retrospective group of indicators, which means that they cannot predict the date of the ovulation before it occurs, they are only able to confirm (to some extent) that ovulation has occurred in the recent past. Although their effectiveness is quite high (especially when using two methods at the same time), the aforementioned inconveniences amount to serious drawbacks of these methods.

Another group of ovulation indicators is based on LH hormone concentration $[8,15]$. There are many different urine or saliva tests and fertility monitoring devices that measure LH. These methods are able to predict ovulation 24 to $72 \mathrm{~h}$ in the future, unfortunately without specifying exactly which of the upcoming days is the most likely to be the ovulation day. Such tests operate by checking whether the given average concentration threshold calculated for the population is exceeded. Therefore it may happen, when someone's usual concentration varies from the norm, that the test will give incorrect results. Disadvantages of these devices include difficulties with interpretation of the results and consequent discrepancies, as well as difficulties with determining the appropriate period in which they should be performed. In addition, the need to buy test strips can often discourage women from using them. Nevertheless, they have the desirable feature of prediction before the actual occurrence of ovulation. 
In order to be able to analyze women's menstrual cycles and to draw conclusions that facilitate detection of ovulation for more general purposes and in other future situations, it is necessary to collect large amounts of data from women with diverse fertility characteristics: e.g. age, occupation, lifestyle, weight, etc. Such data is collected by specialized portals that allow women to record their fertility parameters and provide other services to help couples plan their family. One of them is ovufriend.pl ${ }^{1}$, the leading portal in Poland dealing with this topic. The previous publication presents the architecture of information processing and of its effective storage developed for this portal. The central point of the system is a data warehouse that provides data storage services in the sense of Big Data.

The purpose of creating a new ovulation detection algorithm, is to address the need for a universal, easily accessible solution that abolishes or reduces the existing barriers for users. Additionally, it is to use the existing knowledge and science, skillfully adjusting it to fit the real-life situations (e.g. combining fragmentary information obtained with various ovulation detection methods) and to support new methods of automatic data acquisition, e.g. wearable devices like fit bracelets $[4,7,8]$. The solution is also meant to be effective by economically processing large amounts of data on different scales and from many diverse users that represent different features present in the population. This solution utilizes data of many women to learn possible solutions for currently processed cycles (by computing complex multi-aspect similarities to other cycles). It creates the possibility of forecasting (determining in the future) the date of ovulation, as opposed to only confirming its occurrence in the recent past. This last point is the most important, because it is a kind of breakthrough in the current approach to fertility designation in the home environment. To achieve this goal, methods of artificial intelligence known as similarity-based reasoning were applied.

This paper is an overview of the solution that was developed for a commercial application. For this reason the details of the implementation are omitted and the focus is on the ideas behind the solution. The paper is organized as follows. Section two describes the data collected and its interpretation as compound objects. Sections three and four respectively describe solutions and methods applied during the process of research and development of the novel method of forecasting the date of ovulation. Section five provides evaluation results for different phases of the menstrual cycles. The last section provides elements of discussion and concludes our article.

\section{Menstrual Cycle as a Compound Object}

The central object of interest is the menstrual cycle described by the ensembles of time series inter-correlated with each other, constructed from observations taken by women. The individual components are indexed with the same time quanta representing particular days of the cycle. Depending on the cycle and the woman's behavior there are many possible combinations of data types that

\footnotetext{
${ }^{1}$ https://www.ovufriend.pl.
} 
constitute this multivariate time series [2]. The set of features contains: BBT, cervical mucus, cervix parameters, LH urinary tests, pregnancy tests, statistics and occurrence of user-specific symptoms that may signify approaching ovulation.

$B B T$ data consists of temperature values. The measurements are compared to the mean temperature of the previous 6 days. At the same time other factors are computed (eg. mean, relative difference, etc.) and stored together with the BBT time series for later processing.

Cervical mucus is defined by one of five possible values taken from the enumerative scale: dry, sticky, creamy, watery, stretchy. Each value describes different state of the mucus. Making use of this parameter requires detection of patterns in its variability. Therefore it is not enough to get a single measurement. The data should be collected day by day in a certain range.

Cervix has three parameters that can be tracked: opening, position and texture. Each of them has three values respectively: \{open, medium, closed $\}$, $\{$ high, medium, low $\}$, \{soft, medium, hard\}. The observations are collected independently, but the interpretation of the whole state depends on all these values combined (at least two of them). These data create an additional nested three dimensional time series that describes one feature.

Ovulation test has a binary value: positive or negative. However there are some difficulties with interpreting its result which sometimes leads to wrong classification as one of these two states on part of the user. In this type of data a series of measurements is also required, in particular one containing a transition from negative to positive values. A single positive measurement is often not enough to accurately determine ovulation day.

Pregnancy test also has binary positive and negative values. If a woman got pregnant during the cycle, the pregnancy test will come out positive, but only if it was taken an appropriate amount of time after the ovulation. Thus both positive and negative values of pregnancy test in such a cycle may convey some information on the date of the ovulation.

Statistics are useful, because the length of the luteal phase is expected to be constant across a given woman's menstrual cycles. Simple statistical data particular to the user are used: average cycle and luteal phase lengths.

Symptoms are the most complex feature in terms of stored information. It consists of more than 80 elements which describe symptoms (e.g. various pains, mental states, infections, libido, etc.) on a single day of the cycle. Most of them are binary, but together they create a complex structure. Elementary symptoms are granulated and combined into groups of similar elements.

A representation of a single menstrual cycle can be any combination of these data. Moreover, each of the time series independently may require handling of missing values and of imprecision of processed values [5]. An important element is a correlation of the particular sub-time-series. Thus there arises the need to create a representation whose values are determined by the mutual influence of the individual parts of the multivariate time series. 
Such combined time series for each cycle constitutes a compound object described by various features and consisting of many sub-objects in the sense of the definition in [12]. Having a compound object described in this way, allows for the use of the methodology of a network of compound object comparators $(\mathrm{NoC})$ as a tool to perform reasoning based on similarity [12].

\section{$3 \quad$ Fuzzy Networks of Comparators (NoC)}

Networks of Comparators (NoC) are described in detail in [11] and [12]. The reader interested in theoretical or practical aspects of NoC like precise mathematical formulas or training methods should consult these sources. NoC is a general approach to reasoning about compound objects based on their multiaspect similarity to other objects. In a sense $\mathrm{NoC}$ models analogies between objects and their mutual relations. It is most useful in situations that involve information granularity (see [13]). NoC's operation can be interpreted as the calculation of the following function:

$$
\mu_{\text {net }}^{\text {ref }_{\text {out }}}: X \rightarrow[0,1]^{\mid \text {ref }_{\text {out }} \mid},
$$

where the argument is the input object $x \in X$ and ref $_{\text {out }}$ is the reference set for the network's output layer. The target set (co-domain) of $\mu_{\text {net }}^{\text {ref }_{\text {out }}}$ is the space of proximity vectors. These vectors encapsulate information about similarities between the given input object $x$ and objects from the reference set ref $\mathrm{out}_{\mathrm{ou}}=$ $\left\{y_{1}, \ldots, y_{n}\right\}$. The value of the network's function is given by formula

$$
\mu_{\text {net }}^{\text {refout }}(x)=\left\langle\operatorname{SIM}\left(x, y_{1}\right), \ldots, \operatorname{SIM}\left(x, y_{n}\right)\right\rangle,
$$

where $\operatorname{SIM}\left(x, y_{i}\right)$ is the value of global similarity established by the network for the input object $x$ and a reference object $y_{i}$. Global similarity depends on partial (local) similarities calculated by the elements of the network: layers, comparators, local aggregators, translators, projection modules and global aggregators.

In the NoC there are three types of layers: input, intermediate and output. The similarity functions for each type of layer are denoted respectively by $\mu_{\text {layer-in }}^{\text {ref }}, \mu_{\text {layer-int }}^{\text {ref }}$ and $\mu_{\text {layer-out }}^{\text {ref }}$, where ref $_{i}$ is the reference set corresponding to layer $i$. Further algebraic details are provided in [12]. A given network may have several intermediate layers. Each layer consists of comparators that are grouped together by the common purpose of processing a particular piece of information (attributes) about the object in question. Each layer contains a set of comparators working in parallel and a specific translating/aggregating mechanisms that facilitate the flow of information (proximity vectors) between layers. As sets of comparators in a particular layer corresponds to a specific combination of attributes and each returns own similarity vector, local aggregator responsible for converting a similarity matrix into one vector for whole layer is needed. If reference sets are different in consecutive layers, then $t$ the output of 
the previous layer has to be translated first. Additionally a projection module can be used in a layer if there is a need to select a subset of coordinates of a proximity vector to be preserved (e.g. above a threshold, top N, etc.).

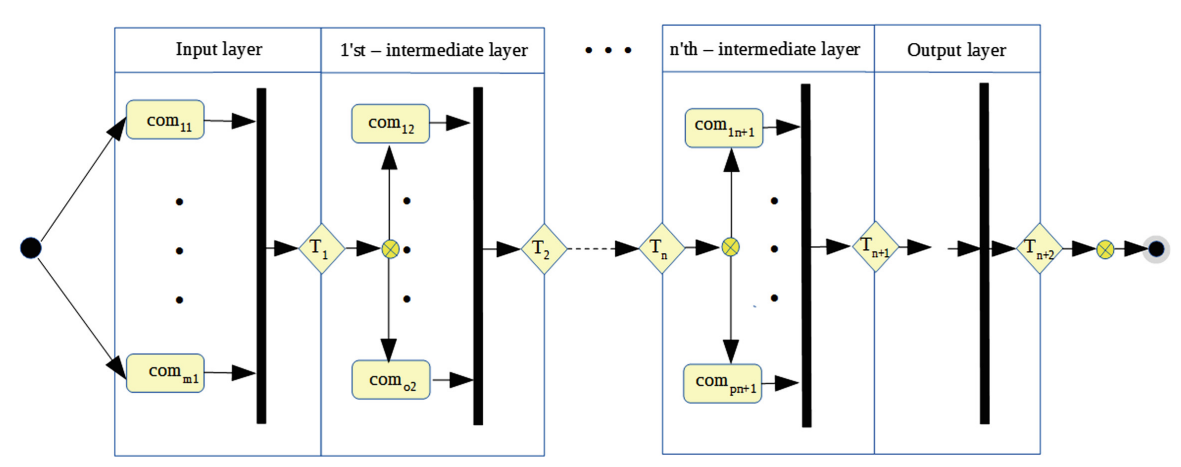

Fig. 1. General scheme of a comparator network in UML-like representation. Notation: com $_{i j}$ - comparators, $\mathrm{T}_{i}$ - translators. Symbols: oval - comparator, thick vertical line - aggregator, rhombus - translator, encircled cross - projection module.

The global aggregator is a compulsory element of the output layer. Unlike local aggregators, which process results within a single layer, the global aggregator may process values resulting from all layers at the same time. This element implements methods of consensus reaching [6]. The comparator network function can be expressed as a composition of functions from subsequent layers:

$$
\mu_{\text {net }}^{\text {ref }_{\text {out }}}(x)=\mu_{\text {layer-out }}^{\text {ref }_{\text {out }}}\left(\mu_{\text {layer-int }}^{\operatorname{ref}_{k-1}} \ldots\left(\mu_{\text {layer-in }}^{\text {ref }_{1}}(x)\right) \ldots\right) .
$$

The graphical interpretation is presented in Fig. 1.

The final result of the NoC is fuzzy set, that's why there is required a defuzzificator to be used, to get crisp result at the end. This element for NoC was described in details in [14].

\section{Proposed Solution}

The designed solution is based on multistage classifiers that accumulate information throughout multiple menstrual cycles. A given cycle is thus important not only for its duration, but it also provides ovulation prediction in the user's future cycles. Multistage classifier is a general concept that describes processing information in parts and in a time sequence.

The algorithm consists of a set of sub-algorithms (called detectors) that independently analyze the available information about the menstrual cycle. They are divided into two classes - prognostic and retrospective. Each of them tries to detect clues to the occurrence of ovulation contained in the type of information that it processes. Every detector returns a fuzzy set whose universe is the set of 


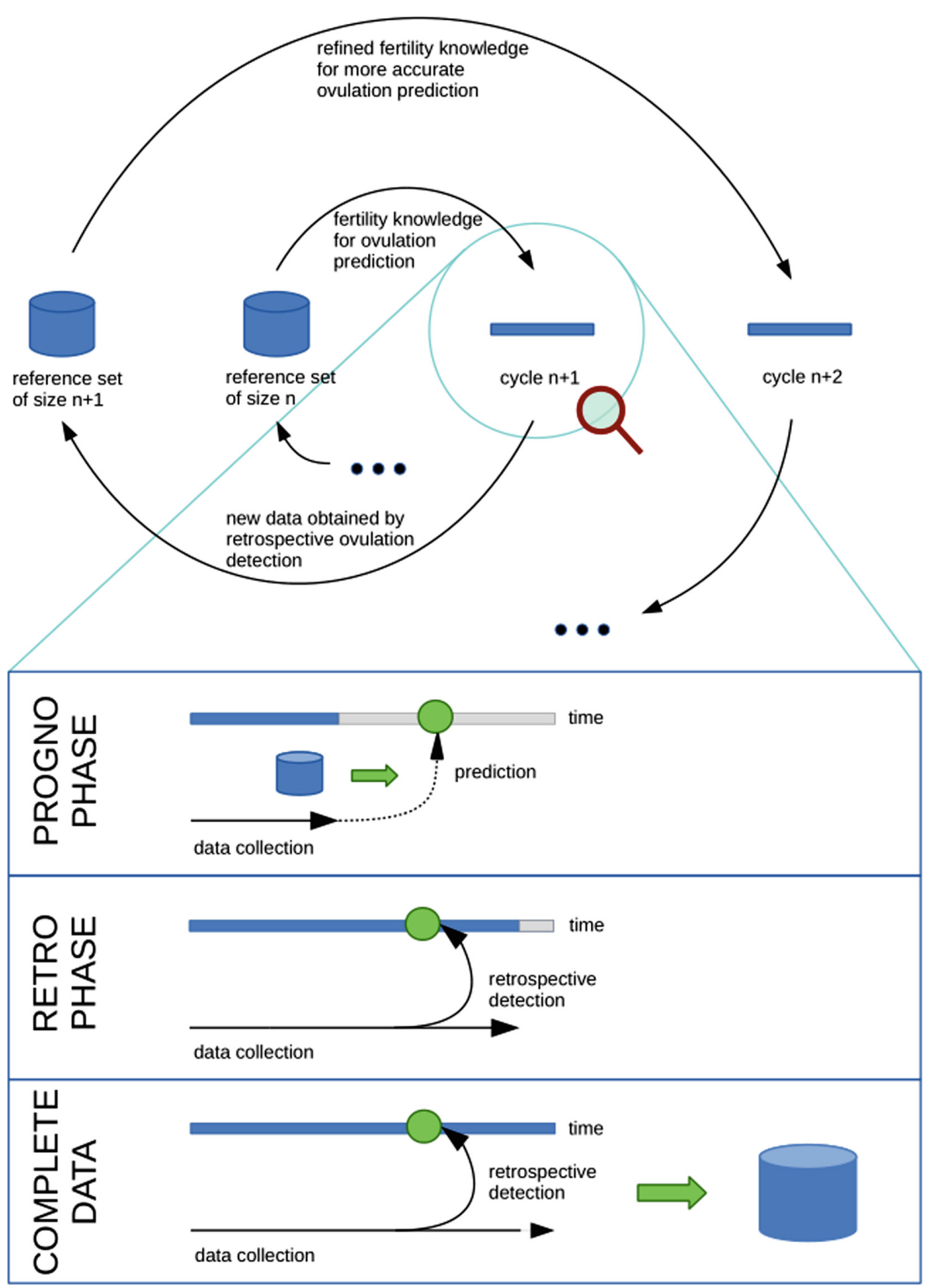

Fig. 2. General scheme of ovulation window prediction algorithm. Upper part presents general look at the data flow process in the algorithm which is arranged in the form of a spiral, where data is processed repeatedly in subsequent iterations on subsequent levels. The bottom part shows details of ovulation detection divided into progno and retro phases. 


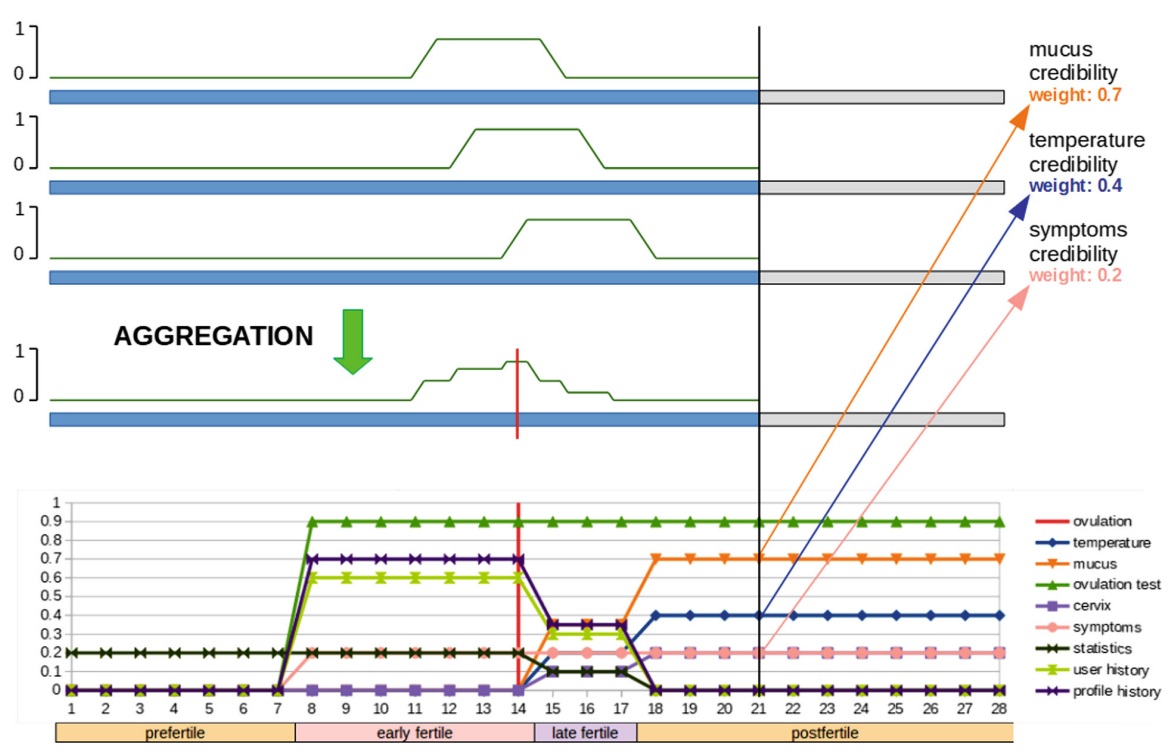

Fig. 3. As time progresses, the cycle advances through four successive sub-phases. Each of them has a different set of weights that determine the impact of the results of different detectors on the aggregated result. In this example results of three detectors are aggregated in the postfertile sub-phase. The red line marks the day to which the algorithm assigned the highest credibility of ovulation. (Color figure online)

all days of the cycle. This fuzzy set describes the ovulation process where the value of the membership function on a given day, represents the credibility that in given day the ovulation exists. The outputs of all detectors are aggregated to obtain a single fuzzy set. The aggregation process is dependent on the current phase of the ongoing cycle. The two main phases of ovulation detection during the cycle are the prognostic phase and the retrospective phase. They are subdivided into four sub-phases: prefertile, early fertile, late fertile and postfertile. Each is characterized by different weights given during aggregation to the particular detectors of prognostic and retrospective classes (including a zero weight which disables a detector), so that the ovulation detection is catered to the specific sub-phase. In the successive sub-phases the following approaches are realized respectively: purely statistical prediction, prediction based on fuzzy NoC, mix of predictive and retrospective methods and finally only retrospective detection. The weight of a detector during aggregation can also be decreased if data of the particular type is found to be of poor quality (inconsistent, unbelievable) in the given cycle. The process of aggregation is presented in Fig. 3. The aggregated fuzzy set is subjected to some additional processing to filter out improbable days. The analysis and defuzzification of this fuzzy set results in the final crisp decision of the algorithm. There can be used various defuzzifiers but here in this paper the the last max was used. The decision is one of the possible outcomes: the cycle is ovulatory and the algorithm designated the most probable day (or 
possibly several days in the prognostic phase) that the ovulation occurred on, the cycle is anovulatory (this can be decided only in the retrospective phase) or the analysis was inconclusive. The last outcome can happen due to insufficient or inconsistent information provided by the user. Many parameters that steer the decision-making in the algorithm are learned and others are set according to expert knowledge.

The retrospective phase occurs after ovulation has already happened and the algorithm, from the perspective of the end part of the cycle, aims to designate the most probable day of ovulation or to detect that the cycle is anovulatory. The requirement is that the user provided appropriate amount of information throughout the cycle. The algorithm in this phase makes use of the classical retrospective methods of ovulation detection based on analysis of time-series of different attributes like temperature, cervical mucus, state of the cervix, ovulation test results or subjective symptoms. The algorithm implements these methods in form of the fuzzy process and adds to them learning of various parameters. The methods analyzing these different attributes are independent of each other, so the user does not have to collect data on each of them, although the more information the user provides, the more accurate results may be expected. Some gaps in the available information can be reliably filled and the algorithm does that during the preprocessing step. The strength of the retrospective approach is its high efficacy under condition of access to reliable and fairly complete data on the menstrual cycle. It allows to designate ovulation in past menstrual cycles collected in the data base to obtain a vast reference set that makes it possible for the prognostic detectors to perform ovulation prediction in ongoing cycles.

After completing first menstrual cycle the system has some information about the user's fertility. At this stage the real prognostic phase of ovulation detection is enabled. In the prognostic phase the algorithm aims to indicate a future day or a few days when the ovulation will most probably occur. The ovulation prediction is achieved using the fuzzy NoC approach. The network structure is related to the set of attributes that describe the menstrual cycle. It has three layers and it compares time series of five types of data between the current cycle and the historical reference cycles using dedicated similarity measures. Comparisons are made for the time series of BBT, mucus type, cervix state, ovulation test results and symptoms. The algorithm uses various time series distance measures, such as DTW, LCSS and other ovulation case specific ones [10]. The two main prognostic detectors use the same structure of the network, but they work on different reference sets. One of them uses as the reference elements all the previous ovulatory cycles of the user. The other utilizes the clustering of menstrual cycles in the database and creating profiles of similar users which are used to construct the reference set. When the reference set is determined, the detector compares the available part of the ongoing cycle to the reference cycles. If enough similarity is found to a reference cycle, then the reference ovulation day influences the fuzzy set which is an output of the detector. In this phase the algorithm does not mark cycles as anovulatory, although the fact that no similar historical cycle that was ovulatory was found can be interpreted as a possible anovulatory 
indication. This approach was used with success during the evaluation of the algorithm performance in the prognostic phase, however the system is designed to promote fertility and discouraging the user from attempting conception by a possibly inaccurate prediction that the cycle is anovulatory would go against its purpose.

There are three more prognostic detectors in addition to those using NoC. The simplest one uses statistics of user cycles (or of population if those are unavailable), i.e. the average cycle length and average luteal phase length. Its limited usefulness is thanks to (relative) constancy of the luteal phase length. Next one is the detector analyzing ovulation test results. It can predict ovulation in the near future based on the pattern of these results. The detector analyzing symptoms reported by the user is the most complex of the three. It relies on the detection of symptom rules periodically performed on the entire collection of menstrual cycles gathered in the data base. A rule is a combination of symptoms observed on a single day. It has a strength value assigned that measures how strong was the correlation between the first occurrence of this particular combination of symptoms in the cycle and the occurrence of ovulation in the near future. Each rule has an associated fuzzy set pattern that, when the rule is triggered on a given day of the cycle, is used to construct a fuzzy set in the neighbourhood of this day - according to the pattern - that conveys to what degree different future days are likely to be the ovulation day. This allows different rules to predict ovulation different amounts of days into the future.

We may now look holistically at the flow of information through the system. When a new cycle begins, the algorithm is in the prognostic phase and it uses the information accumulated throughout the previous cycles to predict ovulation date in the current cycle. At the very beginning of the cycle, the algorithm is in the prefertile sub-phase when the menstruation probably still lasts. In this time when there is virtually no information on the current cycle, the algorithm offers only simple predictions based on statistics of the user (if available) or the population - it gives the range of seven, five or three days (depending on accuracy) where the ovulation will statistically most probably appear. Next, when the cycle enters into the assumed fertile period, the algorithm proceeds to the early fertile sub-phase. Now that there is some information available on the current cycle, in addition to statistics it starts using the ovulation test detector, the symptom rules and the fuzzy NoC to make much more accurate predictions. From the practical point of view this is one of the most valuable functionalities that the system offers, as it gives comfort of better planning for couples hoping for pregnancy. After the gradual accumulation of individual signs of ovulation from retrospective detectors, the algorithm enters the late fertile sub-phase and starts combining prognostic and retrospective methods to indicate the ovulation day. In this period ovulation is in the near past and a quick confirmation (3-4 days) that the forecast from the previous sub-phase was good is possible. Subsequently the algorithm transitions to the postfertile sub-phase which corresponds completely to the retrospective phase. Having data on most of the cycle it can reliably designate ovulation or determine that the cycle is anovulatory. When the cycle 
concludes, it is added to the reference set of closed cycles and it will help refine ovulation prediction for the cycles that will follow it.

In general, this solution assumes that each new cycle feeds the knowledge base extending the system's capabilities. This way it can adapt to new cases and have the ability to predict ovulation for new types of cycles. Also additional cycles coming into the system help dilute the outliers and smooth the distribution of different types of situations so that general trends become more prominent. In this regard it has some analogy to the Kalman filter. The succession of the two phases - retrospective which allows gathering of new information and prognostic which uses that information to make more accurate predictions - in a way creates a spiral through time. This process is presented in Fig. 2.

\section{$5 \quad$ Evaluation and Results}

The data set used in the experiment contains 1097 menstrual cycles. Every cycle in the set was tagged by medical experts with a label signifying occurrence of ovulation or that the cycle is anovulatory. In the former case the experts also selected the day on which ovulation most probably occurred (or several days if the situation was too vague to reliably select a single day). Additionally, the experts recorded their certainty when making a decision (as a number from the unit interval $[0,1])$.

In the data set there are represented cycles with different levels of completeness of data. Those that have a high level of coverage with data points and those that have noticeably less information available. The cycles can also be classified in terms of types of measurements (time series) provided by the user, e.g. cycles with no data on temperature, cycles with no data on ovulation test, etc. This assures that cycles in the data set are diversified and the data set is balanced in terms of different features.

To designate ovulation or to mark the cycle as anovulatory, learning of a set of coefficients is necessary. The ReSample method was used which randomly selects $33 \%$ of cycles from the data set to the training set and the remaining cycles constitute the testing set. This procedure was performed $k$ times and the counts of particular categories (TP, TN, FP, FN) were summed to compute the average efficiency of the algorithm. It was measured for closed cycles, so the ones that are in the post-fertile phase when the learned set of coefficients is used (only in this sub-phase the cycles can be marked as anovulatory). The results of this experiment (ran $k=10$ times) are shown in Table 1.

Table 1. The averaged results achieved for 1097 cycles divided into 2 subsets (learning $(33 \%)$ and testing $(67 \%))$ with $k=10$ repeats.

\begin{tabular}{l|l|l|l|l|l|l|l}
\hline TP & TN & FP & FN & Pr & Re & F1 & Acc \\
\hline 6097 & 402 & 138 & 707 & 0.90 & 0.98 & 0.94 & 0.89 \\
\hline
\end{tabular}


We also simulated the behaviour of the algorithm in the earlier sub-phases: prefertile, early fertile and late fertile. Since these sub-phases do not use the learned coefficients (because it is not needed to decide between ovulatory and anovulatory), these experiments were ran only once on the appropriately modified cycles.

To analyze efficiency of the algorithm in the pre-fertile sub-phase, each cycle was cut to its first 2 days, so to its very beginning. So prepared, the cycles were processed by the algorithm. Its task was to find the interval in which the ovulation will most probably occur if the cycle will turn out to have ovulation.

Table 2. Results for prefertile sub-phase with two types of sets: F - full set (1097), O - only ovulation cycles (1033). Cycles where cut to length of 2 first days. W - window in which the result was considered.

\begin{tabular}{l|l|l|l|l|l|l|l|l|l}
\hline Set & W & TP & TN & FP & FN & Pr & Re & F1 & Acc \\
\hline F & 1 & 488 & 0 & 66 & 543 & 0.88 & 0.47 & 0.62 & 0.44 \\
\hline F & 2 & 687 & 0 & 66 & 344 & 0.91 & 0.67 & 0.77 & 0.63 \\
\hline F & 3 & 823 & 0 & 66 & 208 & 0.93 & 0.80 & 0.86 & 0.75 \\
\hline $\mathrm{O}$ & 1 & 488 & 0 & 2 & 543 & 0.99 & 0.47 & 0.64 & 0.47 \\
\hline $\mathrm{O}$ & 2 & 687 & 0 & 2 & 344 & 0.99 & 0.67 & 0.80 & 0.67 \\
\hline $\mathrm{O}$ & 3 & 823 & 0 & 2 & 208 & 0.99 & 0.80 & 0.89 & 0.80 \\
\hline
\end{tabular}

Table 2 contains results for all cycles in the reference set of cycles tagged by medical experts (both these with ovulation and these that are anovulatory). Of course, since in this sub-phase the algorithm does not mark cycles as anovulatory, all anovulatory cycles were classified as mistakes. Next, the experiment was performed on the reference set with anovulatory cycles removed. In this case, the efficiency results take into account only the actual efficiency for cycles that had ovulation. The results are also contained in Table 2. They are listed depending on the interval width: 7 (window 3 ), 5 (window 2), or 3 (window 1) days. The narrower the interval, the lower the efficiency.

To simulate the early fertile sub-phase, with the assumption that the ovulation is to be predicted before it happens, the cycles were cut to one day before ovulation for cycles with ovulation and to half-length of the cycle for anovulatory cycles. Two experiments were performed whose results are presented in Table 3 . In the first one if the algorithm did not return any prediction, we interpreted it as the decision that the cycle is anovulatory. This interpretation is justified by the fact that these detectors operate on cycle similarity, so not finding any cycle with ovulation that is similar enough to the cycle we are analyzing, indicates that it may be anovulatory. The second experiment was performed only on ovulatory cycles.

The third sub-phase is such that the ovulation has already occurred, but only in the near past. The simulation of this sub-phase was done by cutting the cycle to the fourth day after the ovulation or in the case of an anovulatory cycle by cutting it to five days before the end. In this sub-phase the algorithm uses both the prognostic and the retrospective detectors. Again, two experiments were performed with the same rules as in the previous sub-phase. Their results are presented in Table 3. 
Table 3. Results for early fertile and late fertile sub-phases using two types of sets: F full set, $\mathrm{O}$ - only ovulation cycles. Results for early fertile phase are placed in columns with $*$. Early fertile cycles were cut to the length of one 1 day before and late fertile cut to the 4 days after the ovulation day. In case of no ovulation half of original length or 5 days before end of cycle was set respectively.

\begin{tabular}{l|l|l|l|l|l|l|l|l|l|l|l|l|l|l|l|l|l}
\hline Set & W & TP $^{*}$ & TN$^{*}$ & FP* & FN* & Pr* & Re* & F1* & Acc & TP & TN & FP & FN & Pr & Re & F1 & Acc \\
\hline $\mathrm{F}$ & 1 & 730 & 68 & 1 & 297 & 0.99 & 0.71 & 0.83 & 0.73 & 850 & 65 & 0 & 182 & 1.00 & 0.82 & 0.90 & 0.83 \\
\hline $\mathrm{F}$ & 2 & 836 & 68 & 1 & 191 & 0.99 & 0.81 & 0.90 & 0.82 & 928 & 65 & 0 & 104 & 1.00 & 0.90 & 0.95 & 0.91 \\
\hline $\mathrm{F}$ & 3 & 910 & 68 & 1 & 117 & 0.99 & 0.89 & 0.94 & 0.89 & 973 & 65 & 0 & 59 & 1.00 & 0.94 & 0.97 & 0.95 \\
\hline $\mathrm{O}$ & 1 & 730 & 5 & 0 & 297 & 1.00 & 0.71 & 0.83 & 0.71 & 850 & 1 & 0 & 182 & 1.00 & 0.82 & 0.90 & 0.82 \\
\hline $\mathrm{O}$ & 2 & 836 & 5 & 0 & 191 & 1.00 & 0.81 & 0.90 & 0.81 & 928 & 1 & 0 & 104 & 1.00 & 0.90 & 0.95 & 0.90 \\
\hline $\mathrm{O}$ & 3 & 910 & 5 & 0 & 117 & 1.00 & 0.89 & 0.94 & 0.89 & 973 & 1 & 0 & 59 & 1.00 & 0.94 & 0.97 & 0.94 \\
\hline
\end{tabular}

\section{Summary}

In this paper we presented a novel approach to designating the ovulation day in women's menstrual cycles using standard declarative data, which can be observed at home without specialized equipment. Particularly noteworthy is the fact that the algorithm allows for prediction of the day of ovulation and not only for its confirmation as is the case with most classic algorithms in this area.

The experiment confirmed the very good efficiency of the algorithm in the different phases of the cycle. The average F1 score value for the retrospective phase is 0.94 , and for the earlier phases it is not much lower. The comparison of efficiency results achieved in different phases of the cycle shows that throughout all of the cycle, the algorithm is able to provide valuable information to a woman that is trying to conceive. Even at the beginning of the cycle, the algorithm is able to indicate an interval in which the ovulation is most likely to occur with relatively high reliability. When the cycle advances and the future ovulation gets closer in time, it is able to indicate the correct ovulation day with very high credibility. These results confirm the effectiveness of the solution and in particular of the networks of comparators used here to process such complex multidimensional time series.

The immediate next step in our work will be focused on application of the solution in the Ovufriend platform products, where real-time users will use the implemented algorithms. The presented algorithm will increase the chance of pregnancy even for couples with a narrow fertility window, because it will more accurately determine the ovulation day and, above all, it will predict it and not only confirm.

\section{References}

1. Bablok, L., Dziadecki, W., Szymusik, I., et al.: Patterns of infertility in Poland multicenter study. Neuro Endocrinol Lett. 32(6), 799-804 (2011)

2. Fedorowicz, J., et al.: Multivariate ovulation window detection at OvuFriend. In: Mihálydeák, T., et al. (eds.) IJCRS 2019. LNCS (LNAI), vol. 11499, pp. 395-408. Springer, Cham (2019). https://doi.org/10.1007/978-3-030-22815-6_31 
3. Fehring, R., Schneider, M., Raviele, K., Barron, M.: Efficacy of cervical mucus observations plus electronic hormonal fertility monitoring as a method of natural family planning. Obstet. Gynecol. Neonatal Nurs. 36(2), 152-160 (2007)

4. Goodale, B.M., Shilaih, M., Falco, L., Dammeier, F., Hamvas, G., Leeners, B.: Wearable sensors reveal menses-driven changes in physiology and enable prediction of the fertile window: observational study. J. Med. Internet Res. 21(4) (2019)

5. Kacprzyk, J., Zadrożny, S.: Fuzzy logic-based linguistic summaries of time series: a powerful tool for discovering knowledge on time varying processes and systems under imprecision. Wiley Interdiscip. Rev. Data Min. Knowl. Discov. 6(1), 37-46 (2016)

6. Kacprzyk, J., Zadrożny, S.: Towards a fairness-oriented approach to consensus reaching support under fuzzy preferences and a fuzzy majority via linguistic summaries. In: Nguyen, N.T., Kowalczyk, R., Mercik, J. (eds.) Transactions on Computational Collective Intelligence XXIII. LNCS, vol. 9760, pp. 189-211. Springer, Heidelberg (2016). https://doi.org/10.1007/978-3-662-52886-0_13

7. Shilaih, M., Clerck, V., Falco, L., Kübler, F., Leeners, B.: Pulse rate measurement during sleep using wearable sensors, and its correlation with the menstrual cycle phases, a prospective observational study. Sci. Rep. 7(1) (2017)

8. Shilaih, M., Goodale, B., Falco, L., Kübler, F., De Clerck, V., Leeners, B.: Modern fertility awareness methods: wrist wearables capture the changes in temperature associated with the menstrual cycle. Biosci. Rep. 38(6) (2018)

9. Smoley, B., Robinson, C.: Natural family planning. Am. Fam. Physician 86(10), 924-928 (2012)

10. Soleimany, G., Abessi, M.: A new similarity measure for time series data mining based on longest common subsequence (2019)

11. Sosnowski, Ł.: Framework of compound object comparators. Intell. Decis. Technol. $\mathbf{9}(4), 343-363$ (2015)

12. Sosnowski, Ł.: Compound objects comparators in application to similarity detection and object recognition. In: Peters, J.F., Skowron, A. (eds.) Transactions on Rough Sets XXI. LNCS, vol. 10810, pp. 169-300. Springer, Heidelberg (2019). https://doi.org/10.1007/978-3-662-58768-3_6

13. Sosnowski, Ł., Szczuka, M., Ślęzak, D.: Granular modeling with fuzzy comparators. In: 2015 IEEE International Conference on Big Data, Big Data 2015, Santa Clara, CA, USA, 29 October-1 November 2015, pp. 1550-1555. IEEE (2015)

14. Sosnowski, Ł., Szczuka, M.: Defuzzyfication in interpretation of comparator networks. In: Medina, J., et al. (eds.) IPMU 2018. CCIS, vol. 854, pp. 467-479. Springer, Cham (2018). https://doi.org/10.1007/978-3-319-91476-3_39

15. Su, H., Yi, Y., Wei, T., Chang, T., Cheng, C.: Detection of ovulation, a review of currently available methods. Bioeng. Transl. Med. 2(3), 238-246 (2017) 